\title{
Neonatal jaundice: aetiology, diagnosis and treatment
}

\section{Abstract}

A significant proportion of term and preterm infants develop neonatal jaundice. Jaundice in an otherwise healthy term infant is the most common reason for readmission to hospital. Jaundice is caused by an increase in serum bilirubin levels, largely as a result of breakdown of red blood cells. Bilirubin is conveyed in the blood as 'unconjugated' bilirubin, largely bound to albumin. The liver converts bilirubin into a conjugated form which is excreted in the bile. Very high levels of unconjugated bilirubin are neurotoxic. Phototherapy is a simple and effective way to reduce the bilirubin level. Most term babies have 'physiological' jaundice which responds to a short period of phototherapy, and requires no other treatment. A few babies have rapidly rising bilirubin levels which place them at risk of kernicterus. Current management of jaundice in the UK is guided by the NICE guideline. Any infant with high serum bilirubin or a rapidly rising bilirubin level needs to be treated urgently to avoid neurotoxicity. High levels of conjugated bilirubin in a term baby can indicate biliary atresia, and babies with persisting jaundice must have their level of conjugated bilirubin measured. Preterm infants on long-term parenteral nutrition may develop conjugated jaundice which generally improves with the introduction of enteral feed and weaning of intravenous nutrition.

Keywords: Neonatal jaundice, kernicterus, conjugated jaundice, phototherapy, exchange transfusion

\section{Dr Subhabrata Mitra}

Consultant Neonatologist, Neonatal Unit, Elizabeth Garrett Anderson Wing, University College London Hospital, London NW1 2BU

\section{Dr Janet Rennie}

Consultant Neonatologist, Neonatal Unit, Elizabeth Garrett Anderson Wing, University College London Hospital, London

Correspondence to: Dr Subhabrata Mitra subhabrata.mitra.13बucl.ac.uk
1 eonatal jaundice is one of the most common clinical signs in newborn infants. Jaundice present as yellow discolouration of the skin and sclera in infants, indicating a raised serum bilirubin level leading to accumulation of bilirubin in the tissues, including the skin and mucous membranes. Jaundice is thought to be visible at bilirubin levels of around $90 \mu \mathrm{mol} /$ litre in babies with pale skin tones. The detection of jaundice is more difficult in babies with dark skin tones, but the sclerae are always white and inspection of the eyes is a crucial part of visual assessment of jaundice. Around $60 \%$ of term and $80 \%$ of preterm infants develop jaundice in the first week of life and $10 \%$ of breastfed infants remain jaundiced up to 1 month of age. Jaundice was the most common reason for admission from home to neonatal units in England for term infants (Battersby et al, 2017).

Most infants who become jaundiced develop jaundice in the first week of life, and in the majority of cases it is mild and harmless. Breast-fed infants are more prone to develop physiological jaundice in the first week of life. The key challenge is to differentiate the rare baby with significant jaundice which might lead to bilirubin encephalopathy and kernicterus from the majority of babies in whom jaundice will be harmless. It is also essential to identify infants with conjugated hyperbilirubinaemia who have biliary atresia as early as possible to improve outcome. The outcome for babies with biliary atresia whose surgery is performed at less that 6weeks of age is much better than in those whose surgery is delayed by late diagnosis.

The current national guideline in UK from the National Institute for Health and Care Excellence (NICE) (2016) recommends a review of all infants with risk factors for neonatal jaundice within the first 48 hours of life and assessment of serum bilirubin level in any infant with clinical jaundice. The risk factors are outlined in Table 1.

\section{Billirubin metabolism}

In newborn infants, bilirubin is mostly produced from the breakdown of red blood cells. Haemoglobin, 
released from broken-down red blood cells, undergoes a two-stage catabolism within the reticuloendothelial system to produce bilirubin. This unconjugated (or indirect) bilirubin is transported in the circulation bound with serum albumin. Unconjugated bilirubin combines with glucuronic acid in the smooth endoplasmic reticulum inside the liver to produce water-soluble mono- or di-glucuronides of bilirubin, commonly referred as conjugated (or direct) bilirubin.

Conjugated bilirubin is a component of bile and enters the gut via the biliary system. Although a proportion of conjugated bilirubin can be hydrolysed by beta-glucuronidase to unconjugated bilirubin in the small gut, from where it is reabsorbed (the enterohepatic circulation), the majority is excreted with the stool. Establishing gut motility and a normal pattern of stooling is an important component of reducing the bilirubin load from the enterohepatic circulation in the first few days, which is part of the reason why breastfeeding support is an essential component of the management of neonatal jaundice. An excellent new e-learning resource package regarding physiology of bilirubin metabolism under NHS Health Education England will be available for health-care professionals (http://barnacle.e-lfh. org.uk/elex/repository/MaterialManagement. aspx?_materialId=15591\&rl=prj\&_prjid=15146).

\section{Normal pattern of neonatal jaundice}

Most newborn infants develop jaundice in the first week of life. In term infants, the serum bilirubin level reaches a clinically detectable level by day 3 or 4 . This gradually falls over the next few days before reaching the normal childhood serum bilirubin level by the end of the second week of life. This is generally referred to as physiological jaundice. Although prolonged unconjugated jaundice was previously attributed mainly to 'breast milk jaundice', it is becoming clear that many of these cases have an underlying genetic explanation (Bhutani, 2012). Up to one-third of exclusively breast-fed infants remain jaundiced at the end of 2 weeks and this group contributes to most community referrals to hospital for review at this age. A confident diagnosis of breast milk jaundice can only be made after exclusion of other possible causes and parents need an explanation of the nature of the problem. Beta-glucuronidase is present in breast milk and can cause increased levels of unconjugated bilirubin that enters the enterohepatic circulation from the gut. Altered gut flora in breast-fed infants has also been implicated in the reduction of the conversion of bilirubin glucuronides to urobilinoids.

Jaundice in preterm infants is characterised by a

\author{
Table 1. Factors related to higher susceptibility of \\ newborn infants for development of physiological \\ jaundice \\ High haemoglobin concentration in newborn infants \\ Immaturity of hepatic uptake, transport and conjugation \\ system \\ Shorter lifespan for neonatal red blood cells compared \\ to those of adults \\ Increased level of beta-glucuronidase in the gut, \\ releasing more unconjugated bilirubin to enter the \\ enterohepatic circulation
}

higher peak in serum bilirubin levels along with a longer duration of hyperbilirubinaemia compared to term infants.

Infants with bruising related to birth trauma, scalp haematoma (e.g. cephalhaematoma) and born after delayed cord clamping need to be carefully monitored early on as they have an increased risk of developing jaundice.

Glucose-6-phosphatase deficiency (G6PD) is an important underlying cause of kernicterus, both worldwide and in the UK. Male babies of Asian ethnicity are particularly at risk but this condition is not confined to this group.

\section{Billirubin encephalopathy}

Sick and preterm infants are more vulnerable to the neurotoxic effect of raised bilirubin levels (Gartner et al, 1970). Pathophysiological mechanisms include cell membrane function disruption, lowering of action potential, disturbed energy metabolism and disturbance in neurotransmitter synthesis (Volpe, 2008; Brites et al, 2009). The deposition of bilirubin in the brain, noted during autopsy of infants who had died as a result of acute bilirubin toxicity, was described as 'kernicterus'. Involvement of the brainstem, hippocampus, cerebellum, globus pallidus and subthalamic nuclei has been described. This anatomical predisposition might be related to increased metabolic activity and regional cerebral blood flow.

Hypoxia, acidosis, hypercarbia, sepsis, seizures and disturbances to the blood-brain barrier are co-existing risk factors for acute bilirubin toxicity (Wennberg et al, 2006). Medications that interfere with bilirubin albumin binding or inhibit p-glycoprotein are implicated in increasing the risk of acute bilirubin encephalopathy (Watchko et al, 2002). More apoptosis was noted in less well-differentiated astrocytes and neurons (Brites et al, 2009) indicating 
Table 2. Risk factors associated with the development of kernicterus

Serum bilirubin level $>340 \mu \mathrm{mol} /$ litre in a term infant ( $\geq 37$ weeks)

Rapid rise in the serum bilirubin level $(>8.5 \mu \mathrm{mol} /$ litre) hour)

\section{Clinical signs suggestive of bilirubin encephalopathy}

the importance of the developmental stage of the brain in relation to bilirubin toxicity. A UK surveillance study has reported an occurrence of kernicterus in 1 in 100 ooo live births (Manning et al, 2007).

Risk factors for developing kernicterus are presented in Table 2.

Previously described different stages of bilirubin encephalopathy (Connolly and Volpe, 1990) are now rare in current clinical practice. Opisthotonus (Figure 1) generally indicates neuronal toxicity. Magnetic resonance imaging of the brain is the investigation of choice in suspected cases and presents a characteristic picture in cases of kernicterus (Figure

Figure 1. Typical opisthotonic posture in a baby with neonatal jaundice and acute bilirubin encephalopathy.

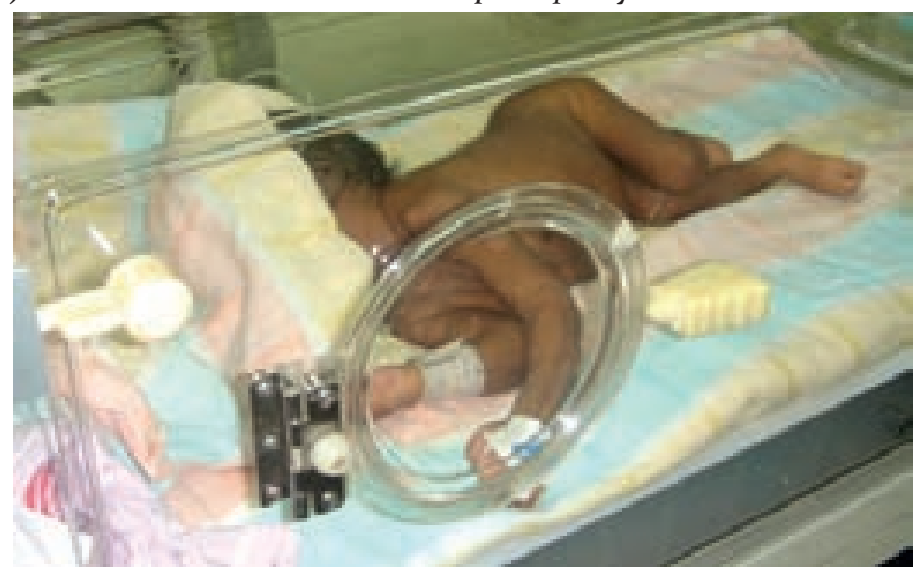

Figure 2. Characteristic changes in kernicterus. $a$ and $b$. Hyperintense signal and symmetrical scarring in globus pallidus on T2-weighted imaging on brain magnetic resonance imaging in kernicterus in two different cases and (c) macroscopic appearance of brain in kernicterus with characteristic yellow bilirubin staining.

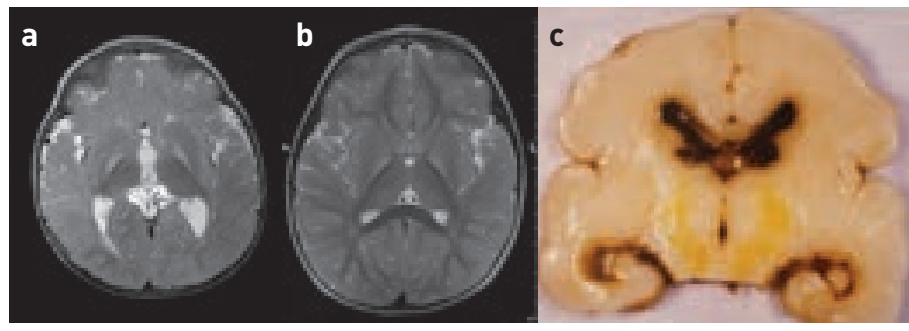

2). High intensity areas in the posteromedial border of the globus pallidus on T2-weighted imaging is the most sensitive finding (Yokochi, 1995; Govaert et al, 2003). It is important to remember that longterm neurological impairment can be associated with normal magnetic resonance imaging after acute bilirubin encephalopathy (Katar et al, 2008). Longterm features include extrapyramidal disturbances, sensorineural hearing loss, auditory impairment and upward gaze palsies. The resultant cerebral palsy normally includes a degree of athetosis.

It is difficult to correlate a specific serum bilirubin level to the onset of bilirubin neurotoxicity. The report from the Pilot USA Kernicterus Registry suggested that a bilirubin level $>598 \mu \mathrm{mol} /$ litre can have a profoundly deleterious effect (Bhutani and Johnson, 2009).

Analysis of the NHS Resolution data regarding settled kernicterus claims in England also found that term babies had bilirubin levels above $600 \mu \mathrm{mol} /$ litre at presentation (J Rennie et al on behalf of the NHS Improvement Patient Safety Programme, 2017, unpublished data).

\section{Aetiology of neonatal jaundice}

In view of the current era of early postnatal discharge and community support in the NHS, risk factors (Khoury et al, 1988; Newman et al, 2002; Keren et al, 2005; Maisels et al, 2009) associated with development of significant jaundice need to be reviewed before discharge (Table 3). The NICE (2016) guideline recommends that a second clinical examination is conducted before 48 hours of age.

A detailed family history and clinical examination is extremely important in all infants with jaundice for understanding of the possible aetiopathophysiology. Appropriate investigations can identify treatable conditions early (e.g. isoimmunisation, infection, biliary atresia) and improve outcome.

\section{Early jaundice}

Clinical jaundice within the first 24 hours of life is likely to be pathological and commonly a result of isoimmunisation (most commonly $\mathrm{ABO}$ or rhesus incompatibility) or other causes of significant haemolysis. Blood group and rhesus status for both mother and infant need to be reviewed along with a direct agglutination test (DAT). Anti-D prophylaxis in rhesus-negative mothers can cause a weakly positive DAT result as a result of passive transfer of antibody. A positive DAT test does not always correlate with the severity of jaundice (Bakkeheim et al, 2009). Reticulocyte count and blood film also carry a low 


\section{Current Issues in Neonatal Care}

Table 3. Risk factors in babies more likely to

develop significant jaundice

Gestational age $<38$ weeks

History of previous sibling needing

phototherapy

Exclusive breast feeding

Clinical jaundice visible within first 24 hours of life

sensitivity and specificity for diagnosis of haemolysis in newborn infants (Newman and Easterling, 1994).

Review of maternal antenatal notes often gives vital information about other blood group incompatibilities (e.g. Kell group) or the presence of other antibodies. Infection should always be excluded in sick newborns with early jaundice. Although G6PD deficiency-related jaundice presents after 48 hours, it needs to be part of the investigations in infants with relevant ethnicity (parents with Mediterranean, Asian or African origin). If haemolysis is ruled out, Crigler-Najjar syndrome should be considered. Although rare, it can develop a rapidly deteriorating non-haemolytic unconjugated jaundice early in life. Infants with G6PD deficiency can also present with non-haemolytic jaundice in the neonatal period.

\section{Prolonged jaundice}

Persistent clinical jaundice in term infants at 2 weeks and in preterm infants at 3 weeks of age is termed prolonged jaundice. This is a common referral from the community and mostly presents as unconjugated jaundice noted in breastfed infants. Feeding history, colour of stool and urine and clinical examination can mostly rule out pathological causes. The investigations should be done stepwise depending on the results from first-line investigations.

Initial investigations for infants with prolonged jaundice:

- Check the colour of stool (whether yellow or pale chalky stool)

- Check the urine (whether it is dark and stains the nappy easily)

- Measure total and split bilirubin

- Full blood count, blood group and DAT

- Liver function test

- Urine culture and sensitivity

- Ensure Guthrie card sent for routine metabolic screening

Thyroid function test

G6PD level.

\section{Conjugated jaundice}

Serum conjugated bilirubin level of $>25 \mu \mathrm{mol} /$ litre is generally considered as conjugated jaundice. Although in clinical practice a $10 \%$ cut-off value for total serum bilirubin level is sometime used, this can give false reassurance in the case of high total bilirubin level. Pale chalky stool and dark urine can be important associated symptoms. Initial investigations should include a liver function test and check for any clotting abnormality. A liver ultrasound can provide further input in cases with suspected obstructive jaundice. Further investigations can rule out congenital infections, sepsis, glucosaemia and aminoacidaemias. Preterm infants on total parenteral nutrition often have a marked increase in conjugated bilirubin fraction which improves gradually after total parenteral nutrition is stopped.

\section{Assessment of neonatal jaundice}

Examination of the newborn for visual assessment of clinical jaundice should be performed in a well-lit room. Visual assessment is unreliable, particularly under artificial light and after phototherapy has begun. It can also be difficult in dark-skinned infants, in whom examination of sclera, gums and pinched skin are also important. For any infant with clinical jaundice, the serum bilirubin level must be measured to allow planning of management. Clinical jaundice usually becomes visually evident at a serum bilirubin level of $80-90 \mu \mathrm{mol} / \mathrm{litre}$ in babies with pale skin.

In recent years, use of a transcutaneous bilirubinometer has transformed community care of jaundiced infants in UK. Bilirubin measurements from these instruments correlate well with serum bilirubin level and can avoid unnecessary blood tests and/or referral to hospital. Although transcutaneous bilirubinometer measurement highly correlates with serum bilirubin measurement, the average difference between these measurements was $12.7 \pm 32.9 \mu \mathrm{mol} /$ litre (Campbell et al, 2011), and this discrepancy increases further above levels of $250 \mu \mathrm{mol} /$ litre. NICE guidance recommends checking a serum sample when transcutaneous bilirubinometer records indicates a bilirubin level higher than $250 \mu \mathrm{mol} / \mathrm{litre}$.

Most neonatal units in UK have the facility to estimate total serum bilirubin level from a bench bilirubinometer using direct spectrometry. For any specific measurement of conjugated and unconjugated bilirubin, a sample should be sent to the hospital biochemistry laboratory.

The investigation pathway for neonatal jaundice (NICE, 2016) is presented in Figure 3. 
AQ please confirm permission from NICE to reproduce this and figure 4 and details of the acknowledgement required?

Figure 3. Investigation pathway for neonatal jaundice. Adapted from NICE (2016) guideline.

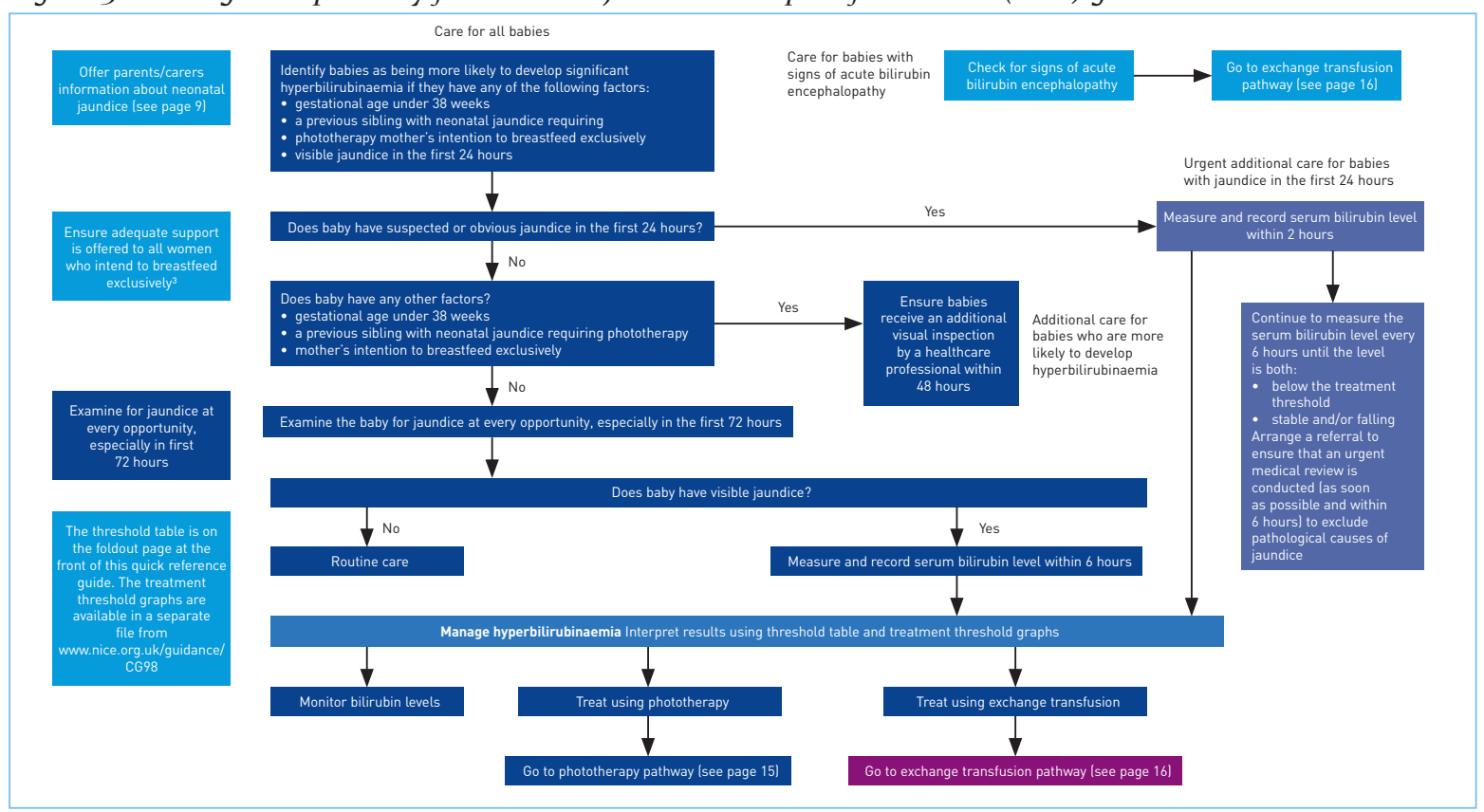

\section{Management of neonatal jaundice}

The management of neonatal jaundice is targeted at identifying the rare baby who has a rapidly rising bilirubin level and is at risk of kernicterus from the majority of term babies whose jaundice will remain harmless. Adequate early support (both pre-discharge and in the community) for mothers and infants for establishment of breast feeding reduces the risk of developing raised serum bilirubin level and can avoid the need for hospital readmission.

\section{Unconjugated jaundice}

\section{Phototherapy}

Phototherapy is the first step in the management of raised unconjugated jaundice in newborn infants. It is a safe and convenient method of lowering serum bilirubin levels and reduces the need for more invasive treatment, exchange transfusion. Phototherapy is effective only after bilirubin enters the skin at serum bilirubin level $>8 \mathrm{o} \mu \mathrm{mol} / \mathrm{litre}$ (Tan, 1982). The efficacy of phototherapy depends on the dose and wavelength of light used as well as the surface area of the infant's body exposed to it. Increasing the dose can be achieved by placing phototherapy units at the minimum safe distance from the infant and increasing the number of units used. The American Academy of Pediatrics has defined the characteristics of a phototherapy device that contribute to the effectiveness in reducing the serum bilirubin level - emission of light in the blue-green range that overlaps the in vivo plasma bilirubin absorption spectrum $(\sim 460-490 \mathrm{~nm})$ and a spectral irradiance of at least $30 \mathrm{microW} / \mathrm{cm}^{2} / \mathrm{nm}$ (Bhutani et al, 2011).

Fibreoptic biliblankets are an effective way to reduce bilirubin level. They help to continue phototherapy during cuddles with parents and can be an effective way to give 'double' phototherapy when used together with an overhead unit. They can be placed in direct contact with the infant as they emit an insignificant degree of heat.

The predominant method of bilirubin elimination is the irreversible photoalteration of bilirubin to a structural isomer called lumirubin, which is a watersoluble compound and is excreted with bile and urine. Two other important pathways of bilirubin photoalteration are photo-oxidation of bilirubin to colourless polar molecules that are excreted in the urine and configurational isomerization of bilirubin isomer to more water-soluble and less toxic isomers (Vreman et al, 2004).

Although phototherapy is generally benign, side effects can include diarrhoea, erythematous rash, increased fluid loss, temperature instability and skin discolouration (tanning and bronze baby syndrome). Improvement of phototherapy systems has dramatically reduced these side effects over the last decade.

The NICE (2016) guideline recommends the use of gestational age-specific treatment threshold graphs for babies with neonatal jaundice which is gestational age specific and gives a clear visual idea about the need for the modality of treatment at start as well as 
the response to treatment. Two clear lines indicate the threshold of phototherapy and exchange transfusion over the first few weeks and days of life. Figure 4 gives an example of the treatment threshold chart for infants $\geq 38$ weeks gestation (NICE, 2016).

\section{Pharmacological agents}

High dose intravenous immunoglobulin is the only pharmacological treatment used in clinical practice for infants presenting with high jaundice levels secondary to rhesus or $\mathrm{ABO}$ isoimmunisation. Although it reduces the need for exchange transfusion, the duration of phototherapy and the length of hospital stay (Gottstein and Cooke, 2003), it is mostly used to buy time before starting exchange transfusion in severe cases of unconjugated jaundice.

\section{Exchange transfusion}

In recent years the need for exchange transfusion has significantly reduced because of improved antenatal management and the availability of improved highly effective phototherapy systems. It is an important intervention for infants who do not respond well to multiple phototherapy and appropriate hydration. It is also indicated in infants born with significant anaemia as a result of in utero haemolysis. In addition to the small risk of blood-borne infection, exchange transfusion carries a risk of morbidity and mortality from vascular injuries, cardiovascular complications, biochemical and haematological disturbance (Keenan et al, 1985). It is vital to monitor the infant closely throughout the procedure and check the haematological and biochemical parameters before, during and after exchange transfusion.

\section{Conjugated jaundice}

Identification of the aetiology is key for the management of an infant with conjugated jaundice. If a congenital obstruction is suspected, the infant needs to be reviewed in a surgical centre for further investigations and management. Biliary atresia is the most common surgically correctable liver condition in this age group and constitutes one fifth of the infants referred to one hepatobiliary referral centre in the UK. In one third of infants with biliary atresia stools are pigmented initially as the intra- and extrahepatic bile ducts may remain patent in the first few weeks of life. As they become atretic, the flow of bile stops and the colour of stool changes to a white, chalky appearance. Success of surgery is highest in earlier infancy, as with time, the intrahepatic biliary ducts obliterate and the chance of success of portoenterostomy drops significantly. Ursodeoxycholic acid (Willot et al,

\section{Key points}

Neonatal jaundice is common in both term and preterm infants

In most cases the jaundice is harmless and requires no treatment or responds to phototherapy

High levels of unconjugated bilirubin can cause permanent brain damage, kernicterus

High levels of conjugated bilirubin can be a marker of biliary atresia, which requires urgent surgery The risk of kernicterus is low but means that all babies with jaundice should have their bilirubin levels monitored closely; babies with risk factors need an evaluation for jaundice at 48 hours.

Figure 4. NICE treatment threshold graph for babies born at $\geq 38$ weeks. Individual charts need to be adjusted according to the gestational age before use.

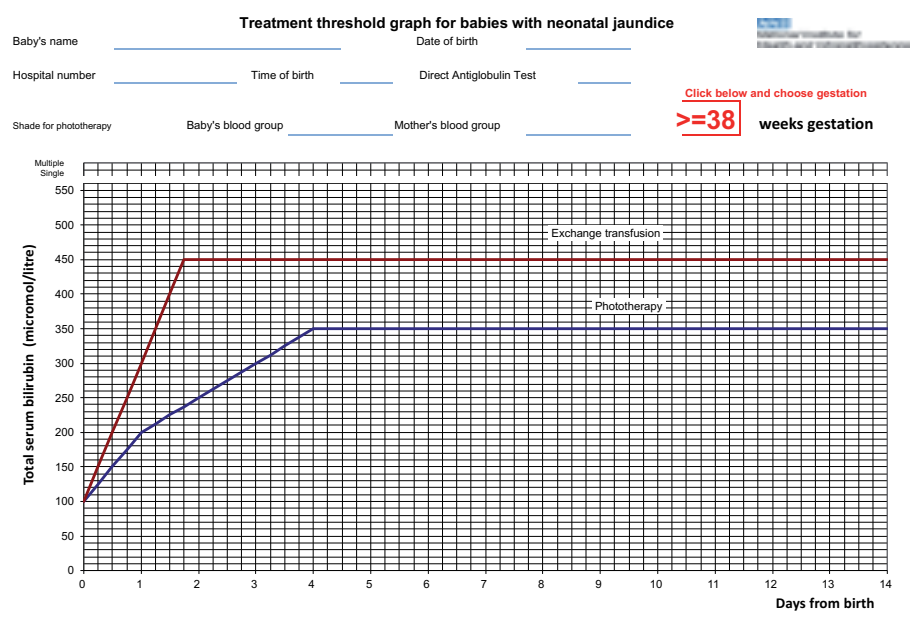

2008) and phenobarbital (Davenport et al, 1997) are used to improve the bile flow, and supplementation of fat-soluble vitamins is also required.

Prolonged use of parenteral nutrition causes cholestasis and hepatocellular damage in early infancy and the incidence increases with the degree of prematurity. Treatment focuses on increasing the bile flow with either ursodeoxycholic acid or phenobarbital and early introduction of enteral feeding.

\section{Conclusions}

Neonatal jaundice is the most common condition needing medical attention in the neonatal period. The majority of these cases present with unconjugated hyperbilirubinaemia and most infants respond well to phototherapy when the bilirubin level reaches the treatment threshold. Infants with risk factors 
for developing significant hyperbilirubinaemia need to be monitored closely and treated early to prevent bilirubin neurotoxicity. Current research may, in future, facilitate the use of the camera facility on mobile phones as an objective measure of the colour of the sclerae (Leung et al, 2015).

Bakkeheim E, Bergerud U, Schmidt-Melbye AC, Akkök CA, Liestøl K, Fugelseth D, Lindemann R (2009) Maternal IgG anti-A and anti-B titres predict outcome in ABO-incompatibility in the neonate. Acta Paediatr 98(12): 1896-1901. https://doi.org/10.1111/j.1651-2227.2009.01478.x

Battersby C, Michaelides S, Upton M, Rennie JM (2017) Term admissions to neonatal units in England: a role for transitional care? A retrospective cohort study. BMJ Open 7(5): eo1605o. https://doi.org/10.1136/bmjopen-2017-016050 Bhutani VK; Committee on Fetus and Newborn; American Academy of Pediatrics (2011) Phototherapy to prevent severe neonatal hyperbilirubinemia in the newborn infant 35 or more weeks of gestation. Pediatrics 128(4): e1046e1052. https://doi.org/10.1542/peds.2011-1494

Bhutani VK (2012) Jaundice Due to Glucose-6-Phosphate Dehydrogenase Deficiency. Neoreviews 13: e166

Bhutani VK, Johnson L (2009) Kernicterus in the 21st century: frequently asked questions. J Perinatol 29: $\mathrm{S}_{20}-\mathrm{S}_{24}$. https://doi.org/10.1038/jp.2008.212

Brites D, Fernandes A, Falcão AS, Gordo AC, Silva RF, Brito MA (2009) Biological risks for neurological abnormalities associated with hyperbilirubinemia. J Perinatol 29 Suppl 1: S8-S13. https://doi.org/10.1038/jp.2008.214

Campbell DM, Danayan KC, McGovern V, Cheema S, Stade B, Sgro M (2011) Transcutaneous bilirubin measurement at the time of hospital discharge in a multiethnic newborn population. Paediatr Child Health 16(3): 141-145.

Connolly AM, Volpe JJ (1990) Clinical features of bilirubin encephalopathy. Clin Perinatol 7(2): 371-379.

Davenport M, Kerkar N, Mieli-Vergani G, Mowat AP, Howard ER (1997) Biliary atresia: the King's College Hospital experience (1974-1995). J Pediatr Surg 32(3): 479-485. https://doi.org/10.1016/Soo22-3468(97)90611-4

Gartner LM, Snyder RN, Chabon RS, Bernstein J (1970) Kernicterus: high incidence in premature infants with low serum bilirubin concentrations. Pediatrics 45(6): 906-917.

Gottstein R, Cooke RW (2003) Systematic review of intravenous immunoglobulin in haemolytic disease of the newborn. Arch Dis Child Fetal Neonatal Ed 88(1): F6-F1o. https://doi.org/10.1136/fn.88.1.F6

Govaert P, Lequin M, Swarte R et al (2003) Changes in globus pallidus with (pre)term kernicterus. Pediatrics $112(6 \mathrm{Pt}$ 1): 1256-1263.

Katar S, Akay HO, Taskesen M, Devecioglu C (2008) Clinical and cranial magnetic resonance imaging (MRI) findings of 21 patients with serious hyperbilirubinemia. J Child Neurol 23(4): 415-417. https://doi.org/10.1177/o88307380730978o

Keenan WJ, Novak KK, Sutherland JM, Bryla DA, Fetterly KL (1985) Morbidity and mortality associated with exchange transfusion. Pediatrics 75(2 Pt 2): 417-421.

Keren R, Bhutani VK, Luan X, Nihtianova S, Cnaan A,
Schwartz JS (2005) Identifying newborns at risk of significant hyperbilirubinaemia: a comparison of two recommended approaches. Arch Dis Child 9o(4): 415-421. https://doi.org/10.1136/adc.2004.060079

Khoury MJ, Calle EE, Joesoef RM (1988) Recurrence risk of neonatal hyperbilirubinemia in siblings. Am J Dis Child 142(10): 1065-1069.

Leung TS, Kapur K, Guilliam A, Okell J, Lim B, MacDonald LW, Meek J (2015) Screening neonatal jaundice based on the sclera color of the eye using digital photography. Biomed Opt Express 6(11): 4529-4538. https://doi. org/10.1364/BOE.6.004529

Maisels MJ, Deridder JM, Kring EA, Balasubramaniam M (2009) Routine transcutaneous bilirubin measurements combined with clinical risk factors improve the prediction of subsequent hyperbilirubinemia. J Perinatol 29(9): 612-617. https://doi.org/10.1038/jp.2009.43

Manning D, Todd P, Maxwell M, Platt MJ (2007) Prospective surveillance study of severe hyperbilirubinaemia in the newborn in the UK and Ireland. Arch Dis Child Fetal Neonatal Ed 92(5): F342-F346. https://doi.org/10.1136/ adc.2006.105361

National Institute for Heath and Care Excellence (2016) Jaundice in newborn babies under 28 days. Clinical guideline [CG98]. https://www.nice.org.uk/guidance/cg98 (accessed 19 October 2017)

Newman TB, Easterling MJ (1994) Yield of reticulocyte counts and blood smears in term infants. Clin Pediatr (Phila) 33(2): 71-76. https://doi.org/10.1177/ooo992289403300202

Newman TB, Liljestrand P, Escobar GJ (2002) Jaundice noted in the first 24 hours after birth in a managed care organization. Arch Pediatr Adolesc Med 156(12): 1244-1250. https://doi.org/10.10o1/archpedi.156.12.1244

Tan KL (1982) The pattern of bilirubin response to phototherapy for neonatal hyperbilirubinaemia. Pediatr Res 16(8): 670-674. https://doi. org/10.1203/00oo6450-198208000-00o18

Volpe JJ (2008) Neurology of Newborn. 5th edn. WB Saunders, Philadelphia

Vreman HJ, Wong RJ, Stevenson DK (2004) Phototherapy: current methods and future directions. Semin Perinatol 28(5): 326-333.

Watchko JF, Daood MJ, Biniwale M (2002) Understanding neonatal hyperbilirubinaemia in the era of genomics. Semin Neonatol 7(2): 143-152. https://doi.org/10.1053/ siny.2002.0102

Wennberg RP, Ahlfors CE, Bhutani VK, Johnson LH, Shapiro SM (2006) Toward understanding kernicterus: a challenge to improve the management of jaundiced newborns. Pediatrics 117(2): 474-485.

Willot S, Uhlen S, Michaud L, Briand G, Bonnevalle M, Sfeir R, Gottrand F (2008) Effect of ursodeoxycholic acid on liver function in children after successful surgery for biliary atresia. Pediatrics 122(6): e1236-e1241.

Yokochi K (1995) Magnetic resonance imaging in children with kernicterus. Acta Paediatr 84(8): 937-939. https://doi. org/10.1111/j.1651-2227.1995.tb13796.x 